\title{
NOTA SÔBRE A FREQÜÊNCIA DO TRONCO BICARÖTICO NO CÃO
}

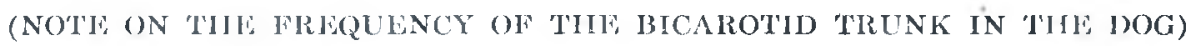

Ortando M. Paiva

Assistente

Fruto de ohscrvaçães registradas, muitas das vêzes, ao curso de trahalhos rotineiros de dissecçĩo escolar, apresentamos os resultados referentes à preparaÇão das colatcrais calibrosas do Arcus aorlicus, no cão doméstico, tendo por intento apurar a freqüência do Truncus bicaroticus. Ao reunir material destinado à realização dêste estudo, tivenos, aliás, oportunidade de assinalar a ocorrência de dois casos de A. subclavia dextra como última colateral do Arcus aorticus, dando-as a conliecer em publicação precedente (PAIVA - 1948), fato que, avaliada a raridade dos achados, dispensa-nos de explicação.

Embora algo se haja escrito no respeitante à variabilidade de comportamento dos ramos oriundos da A. brachiocephalica*, apontando-se mesmo as disposições gerais que as Aa. carótidas primitivas e subclávia direita poderiam assumir no cão, apesar também de dissentirem os tratadistas acêrca da maneira de ramificação das colaterais daquela artéria, no mesmo mamífero, a busca pracedida na bibliografia a nosso alcance, positivou a carência de dados sôbre a freqüência do Tronco bicarótico.

Forçoso é reconhecer, entretanto, a impossibilidade de se levar a efeito, em nosso meio, revisão completa da literatura, para excluir, decisivamente, a existência de eventual nota anatômica mencionando a variedade em estudo; por outro lado, é inquestionável também que, a ocasional observação de casos de origem das Aa. carótidas primitivas por tronco comum, associada a outros aspectos inusitados do sistema arterial —o suficiente importantes para ocultar, sob dife. rentes títulos, a anotação de menor significado - terá contribuido, em muito, para apoucar o valor da pesquisa bibliográfica empreendida.

* A A. brachiocephalica é também denominada A. anonyma, Truncus anonymus e Truncus brachiocephalicus, tendo esta última designaçĩo, proposta pela I.N. A., merecido a segruinte referincia por parte de STIEv (1939): "lìne sinngemässe Bezeichnung für die arteria anonyma der I3.N.A., sie wird in der vergleichenden Anatomie schon lange gebraucht"; "upesar disso, quer em tratados, quer em trabalhos especiais publicados recentemente - e o de nossa autoria, ja citado, está no caso - tem-se preterido o têrmo $T$. brachiocephalicus as de A. brachiocephalica. 
Concernentes ao comportamento dos ramos mais calibrosos do Arcus aorticus no cão, resumimos, nas linhas seguintes, os pareceres dos tratadistas; quase todos admitem que:

1) da crossa aórtica se originam dois ramos, a saber: a) A. brachiocephalica e b) A. subclavia sinistra;

2) da A. brachiocephalica partem: a) A. carolis contmunis sinistra; b) A. carotis communis dextra e algumas vêzes também c) A. thyreoidea caudalis; depois de emitir êsses vasos a $A$. brachiocephalica passa a denominar-se $A$. subclavia dextra;

3) as Aa. carotides communes sinistra e dextra, nascem isoladamente, da A. brachiocephalica, sem formar, portanto, Truncus bicaroticus.

De fato, assim o entendem Ellenberger-Baum - Anat. Descr. e Top. do Cão - $(1891)^{*}$, Lesbre (1923), Mannu in Zimmerl (1930), Euli.enberger-Baum (1932), Martin (1923), Sisson-Grossman (1945), Miti er -- Guia para a dissecção do cão - (1947), Bruni-Zimmelr (1947), Bradley-Grahame Anat. Top. do Cão - (1948), ao passo que LuCarelli (1893), citado por Mobilio (1910), Bossi in Bossi-Caradonna (1909), Gonzálliz y Garcia-Ái varfiz (1929) opinam diferentemente. Com efeito, pretendem os AA. dos dois últinıs tratados conformar a maneira de emergência das AA. carótidas dos carnívoros à dos suídeos; ora, enquanto Bossı assinala a ocorrência de dito tronco nos carnívoros e suínos, já González y Garcia-Álvarez declaram e ratificam a idéia de que as Aa. carótidas comuns dos carnívoros "nascem como no porco com independência".

A rigor, tanto a afirmação de Bossi quante a de Gonzálezz y CARcis-Á vatezz são inexatas, porquanto, pretendem supor idêntico o modo de origem das Aa. carótidas comuns de carnívoros e suínos, quando é exatamente a ausência do Tronco bicarótico nos primeiros e sua presença nos segundos que se constituem, em condiçöes normais, nos caracteres diferenciais precípuos dos ramos da A. braquiocefálica dêsses mamíferos domésticos.

Gonzádez y Garcia-Álvarez, no entanto, e por curioso que pareça, mediante duas afirmativas incorretas, a saber: 1) as $\Lambda$ a. carótidas dos carnívoros nascem como no porco; 2) as Aa. carótidas dos suínos desprendem-se, isoladamente, do Tronco braquiocefálico, levam-nos à seguinte conclusão precisa: as Aa. carótidas dos carnívoros nascem com independência...

* Embora os AA. não declarem no texto de seu tratado, que as Aa. carótidas primitivas partem isoladamente da $A$. hraquiocefálica, representam de modo inequívoco tal disposição, ao ilustrar, en esquema, as artérias da cavidade torácica. 
Quanto a LuCARFLlı, referindo-se à A. braquiocefálica, escreve: "Dal tronco il primo ramo a spiccarsi è la carotide sinistra, como si vede in alcune scimmic, nei putorii, nella tigre, nel coniglio, nella cavia, nello scoiattolo, nel maiale e nel pangolino; oppure si diparte un tronco comune alle due carotidi, $e$ ciò se osserva nel leone, nel gatto, nella volpe, nel cane, nell'orso, nella lontra, nel musoragno, nella giraffa e nei canguri".

Haveria ainda que acrescentar à lista de tratadistas, FAvir.LI (1931), cuja opiniāo, todavia, transcrevemos em separado, por nāo ser possível filiá-la a um dos grupos apontados. Com efeito, êsse A. limita-se simplesmente a apontar a procedência de ambas as Aa. carótidas do "tronco braquial direito", nos carnívoros, suínos e roedores, sem aludir à constituição do Tronco bicarótico.

Ocupemo-nos, agora, das publicações especiais, antecipando ressentirem-se as apreciações nelas contidas da expressão que só a pesquisa sistemática lhes emprestaria. Como se verá, são meros e fortuitos relatos que, embora pouco numerosos, são, mesmo assim, também discordantes.

Moвilio afirma ter notado que, no cão, o tronco das Aa. carótidas comuns pode faltar; nestes casos, o Tronco braquiocefálico fornece primeiramente a $\mathrm{A}$. carńtida esquerda, depois, a distância que pode atingir $16 \mathrm{~mm}$, a A. carótida direita e enfim a A. axilar direita. Em outros casos, o Tronco braquiocefálico divide-se ao mesmo nivel nos três ramos terminais.

Brandt (1940), ao registrar dois casos de Arco aórtico direito, com decurso anormal da A. subclávia esquerda, explica: de hábito, no cāo, o Arco aórtíro dá apenas dois ramos, a saber, A. braquiocefálica e a $\mathrm{A}$. subclávia esquerda. A primeira origina-se aproximadamente da metade do arco, dirige-se cranialmente, cmite as Aa. carótidas comuns esquerda e direita e depois prossegue como A. subclávia direita. O A. omite, em sua exposição, ao apresentar as observa. çồ necroscópicas, o comportamento das Aa. carótidas comuns no tocante à sua origem, sendo de se supor, portanto, tenham a emergência reconhecida como usual.

Davies e Ottaway (1943) afirmam, ao discutir caso de obstrução do esôfago, no cão, determinada por anomalia congênita do Arco aórtico, que, em condiçōes normais, o primeiro ramo da crossa é o Tronco braquiocefálico. Este, origina-se da parede dorsal da aorta, passa obliquamente através da linha mediana, sob a traquéia e esôfago, para se continuar como A. axilar direita, dando primeiı a $A$. carótida cervical esquerda e depois a $A$. carótida cervical direita, quando passa a linha da traquéia e esôfago. A A. axilar esquerda parte imediatamente para trás da direita, também da superfície dorsal da aorta.

No espécime motivador da publicação, reportam os AA., o primeiro ramo a originar-se da crossa, aproximadamente a $2.5 \mathrm{~cm}$ do coraçāo, era curto Tronco 
bicarótico. Este logo se dividia, dando saída às Aa. carótidas cervicais direita e esquerda, normais. 0 ramo seguinte, a A. axilar direita, partia imediatamente para trás do Tronco bicarótico, enquanto a A. axilar esquerda emergia cêrca de $2.5 \mathrm{~cm}$ além do vaso homônimo direito.

PaIva, apresentando dois casos de A. subclavia dextra como última colateral do Arcus aorticus, no cão, descreve sucintamente a disposição tida como normal para os carnivoros domésticos, no tocante aos ramos conspícuos da crossa aórtica: dois colaterais são fornecidos, a $A$. brachiocephalica e a $A$. subclavia sinistra; a $A$. brachiocephalica, por seu turno, emite, sucessivamente, as Aa. curotides communes sinistra e dextra, continuando-se, então como A. subclavia dextra.

Os exemplares que deram causa ao relato de ocorrências julgadas pouco co. nhecidas no cão, mostravam, como primeiro ramo calibroso aúrlico, o tronco das Aa. carotides communes sinistra e dextra, ao qual se seguiam, em ordem, a curta distância, as origens das Aa. subclaviae sinistra e dextra.

Como complemento a estas nótulas bibliográficas, não poderíamos preterir o importante depoimento do Prof. Malcolm E. Miller * do Dep. Anat. -- N.Y. Sta. Vet. Coll., por sabê-lo empenhado na revisão do sistema arterial do cão. Ei-lo: "I have seen three dissections containing truncus bicaroticus in the nearly 500 dissections I have made or observed".

\section{MATERIAL E MÉTODO DE ESTUdO}

O material de estudo compõe-se de animais adultos e fetos de têrmo, provenientes, na maior parte, os primeiros, do Depósito Municipal da Prefeitura de São Paulo, os segundos, do Departamento de Cirurgia da Faculdade de Medicina Veterinária da Universidade de São Paulo. Abrangendo 139 exemplares, dos quais 89 machos, 41 fêmeas e 9 de sexo ignorado**, recolhidos ao Departamento de Anatomia a partir do $20^{\circ}$ semestre de 1946 até fins de 1950 , excluindo-se o período correspondente ao ano letivo de 1948, destinando-se quase todos aos trabalhos escolares de dissecção, os exemplares adultos compreendiam 72 machos, 19 fêmeas e 4 de sexo não determinado, enquanto 17 machos, 22 fêmeas c 5 outros de sexo desconhecido, compunham o grupo de fetos de têrmo.

Excetuando-se as obs. de n.os 75 (Airdale, macho), 79 (Doberman, macko) e 120 (Dinamarquês, fêmea), as restantes baseiam-se no exame de animais hastardos, em sua maioria machos e adultos, sendo a distribuição dos últinos, no tocante à idade, amplamente variável.

* $\Lambda$ gradecemos ao Prof. Malcolm L. Miller por ter correspondido di solicitaçio que lhe fizemos, em carta, com o objetivo de vir a conhecer o registro, en scus preparados, de casos de origem das Aa. carótidas primitivas, por tronco.

* Fistas observações correspondem a peças reduridas, nas quais não encontramos elementos para especificação do sexo. 
Para dissecção das colaterais conspícuas da crossa aórtica. realizada quer em peças frescas, quer em peças fixadas pelo formol, valemo-nos da técnica de rotina. Em casos especiais, isto é, quando a simples dissecção não permilia elucidar se a origem das Aa. carótidas comuns se fazia muito vizinha ou mediante curtíssimo tronco, removíamos do tórax o coração, acompanhado dos vasos da hase seccionados a distância conveniente, procedendo à abertura e exame da superfície interna da $\mathrm{A}$. braquiocefálica ao nível da emergência dos vasos em questão.

Deixamos de tomar mensurações por julgá-las de pouco significado, dada a natureza do material de estudo; por outro lado, o exame das relaçóes nada ofereceu digno de registro, desde que, presente o Tronco bicarótico, a posição de origem da A. carótida comum esquerda é escassamente modificada.

\section{RESULTADOS}

No material reunido para estudo, o Truncus bicaroticus foi anotado cinco vêzes, quatro em machos (obs. 5-9-14-65) e uma em fêmea (obs. 128); excetuando-se uma, correspondente a feto de têrmo (obs. 65), as restantes recaíram em animais adultos.

Em três casos, uma fêmea (obs. 54) e dois machos (obs. 104 e 135), todos adultos, a saída das Aa. carótidas comuns e subclávia direita, verificando-se à mesma altura, dava à A. braquiocefálica aspecto de trípode, enquanto em dois outros: obs. 35, fêmea, adulta, e obs., 66, fêmea, feto de têrmo, o Truncus bicaroticus estava presente, mas, como disposição associada e conseqüente a anoma. lias de origem e de decurso da $A$. subclavia dextra (vide pág. 274).

Destaque-se ainda que as obs. 65 e 66, correspondentes a fetos de têrmo, macho o primeiro, fêmea o segundo, componentes do grupo de seis provenientes da mesma cria (ohs. 61 a 66) e retirados mediante cesariana, exibiam, respectivamente, Tronco bicarótico e A. subclávia direita como última colateral aórtica, com trajeto supra-esofágico; quanto aos mais, três machos e uma fêmea, nada, merecedor de registro, ofereciam.

Cremos interessante chamar a atenção para os números referentes aos raros casos de ocorrência de Tronco bicarótico: ao fichar a obs. 14, já havíamos encontrado três das cinco peças, nas quais as Aa. carótidas comuns nasciam por tronco.

\section{COMENTARIOS}

Passando à interpretação dos achados, deveremos obrigatơriamente reportar-nos a algumas fases do desenvolvimento embriológico do sistema arterial dos mamíferos. Parece-nos desnccessário agora referir, pornenorizadamente, como o fizemos $\mathrm{cm}$ trabalho anterior, a propósito da origem da $A$. subclavia dextra como última colateral do Arcus aorticus no cão, tôdas as transformaçóes que levam à disposição definitiva do adulto, pois, a constituição do Tronco bicarótico, no 
cão, presumimos, ocorre quase ao cabo dessas transformações. Queremos, entre. tanto, aproveitar a oportunidade para, ao retomar a descrição que a respeito demos, baseada no esquema de Hochstetter, clàssicamente utilizado para explicação das anomalias dos ramos oriundos da crossa aórtica, modificá-la em alguns pontos, que a tanto nos autoriza o estudo de OrTs Llorca (1933), sôbre o desenvolvimento dos arcos arteriais em embriōes de porco.

Residindo a diferença de comportamento das colaterais da A. braquiocefá. lica do cão e do porco, em condições normais, respectivamente, na origem separada e por tronco das Aa. carótidas comuns direita e esquerda, fomos levados a consultar o trabalho de Orts Lrorca, na pressuposição de que nêle haveríamos de encontrar o mecanismo de formação do Tronco bicarótico, isto é, a fase que, ocorrendo por exceção nos cães, determinaria, nestes animais, a concentração das carótidas.

De fato, deparamos com explicação apenas parcial e a reproduzimos, a seguir, em sumário: a A. carótida comum surgiria em embriões de $9.5 \mathrm{~mm} \mathrm{e}$, parece, constituir-se-ia graças ao crescimento intersticial dos tractos vasculares compreendidos entre os $2 .^{\circ}$ e $3 .^{\circ}$ arcos arteriais; provàvelmente, a fusão dos segmentos proximais dos dois arcos mencionados, também concorreria para a formação da A. carótida comum. Em relação à A. braquiocefálica, cujo aparecimento só se daria mais tarde, ainda uma vez presumivelmente, o crescimento intersticial e a junção dos tractos iniciais dos $40^{\circ}$ e $3 .^{\circ}$ arcos aórticos direitos e mais a do $3 .^{\circ}$ arco esquerdo comporiam o processo de concentração das colaterais do vaso em questão. Caberia à $7 .^{\mathrm{a}}$ artéria segmentar esquerda fornecer a secção inicial da A. subclávia esquerda.

A pesquisa de Orts Llorca levou-o ainda à noção de que a origem isolada da A. carótida comum esquerda, tal como se vê na espécie humana, dever se-ia à presença da Aorta ventral no decurso do desenvolvimento do sistema arterial. Pelo contrário, nos animais que apresentam, quando adultos, as Aa. carótidas comuns direita e esquerda e a $\mathrm{A}$. subclávia direita emergindo do mesmo tronco, a ausência da Aorta ventral, em tôdas as fases de desenvolvimento dos arcos branquiais, seria a causa determinante da perda de independência do segundo daqueles vasos. A concentração das Aa. carótidas e subclávias de ambos os lados, em tronco único, como ocorre nos ruminantes, por exemplo, encontraria idêntica justificativa, como, aliás, foi evidenciado por Golub (1931), em embriōes de boi.

Com efeito, nos períodos iniciais do desenvolvimento, ORTs Llorca pôde comprovar a inexistência das Aortas ventrais e a saida de todos os arcos aórti. $\cos$ de dilatação do Truncus arteriosus, denominada Saccus arteriosus ("Aortensacke"). Note-se, entretanto, que a presença das Aortas ventrais na espérie humana e sua ausência nos animais domésticos, essencialmente - e não a existên. cia do Saco arterioso, assinalado no sistema vasal, tanto do homem (Congdon 1922), quanto no do boi (GoluB), e no do porco (ORTS LloRCA) - explica. 
riam, no entender dêste último A., as diferenças finais de todo o desenvolvimento.

Ora, a propósito das Aortas ventrais, o próprio Concodon, citado também por Orts Llonca, após série de observações, conclui: "However, these considerations certainly offer no justification for the use of the term "ventral aortae" in man, since such vessels are not to be found at any stage of his developmen:", e, mais adiante, ainda a respeito dos mesmos vasos, afirma: "There is no phase of human development, however, in which such vessels exist."

Segundo o parecer de Golub, as diferenças que se observam nos diferentes mamíferos, quando adultos, já se fariam notar durante os estádios „precoces da evolução dos arcos branquiais. Este conceito esclareceria a impossibilidade de se utilizar a disposição esquemática de Hochstetter, sôbre a formação do arco aórtico e colaterais, como base para encaminhar a análise de tôdas as anomalias de origem e decurso daqueles vasos nos animais domésticos. De fato, no conjunto de modificações que constituem o processo evolutivo dos arcos branquiais, a hipótese da passagem por série de transiçôes, dentre as quais a primeira corresponderia ao estádio do homem, a seguinte ao dos carnívoros, e assim por diante, é infundada. Tal opinião é admitida como correta por OrTs Llorca.

Fundamentado nestes esclarecimentos, julgamos poder agora apontar o mecanismo de formação do Tronco bicarótico nos cães. Êle dependeria, é lícito admitir-se, do crescimento intersticial e da junção dos tractos proximais dos $4 .^{\circ} \mathrm{e}$ $3 .^{\circ}$ arcos direitos e do $3 .^{\circ}$ arco esquerdo, como se observa no porco. Sabendo-se que, todavia, em condições normais, a saída das Aa. carótidas comuns se faz, no cão, isoladamente e conhecendo-se, por outro lado, que, por exceção. elas podem originar-se independentemente no porco ${ }^{*}$, importaria inicialmente estabelecer o processo mediante o qual estas disposiçôes se fixariam.

A fucão do $40^{\circ}$ e $3 .^{\circ}$ arcos direitos e do $3 .^{\circ}$ esquerdo, nos suínos, dá-se em dois tempos: inicialmente, juntar-se-iam o $4 .^{\circ}$ e $3 .^{\circ}$ arcos direitos e mais tarde, ao tronco dêstes viria unir-se o $3 .^{\circ}$ arco esquerdo. Não se trata aqui de simples conjectura, pois, no embrião de $16.5 \mathrm{~mm}$, da porção dorsal e cranial do $4 .{ }^{\text {? }}$ arco esquerdo originam-se, muito vizinhos, dois ramos, a saher: o curto tronco das Aa. subclávia e carótida comum direitas e a A. carótida comum esquerda, conforme demonstrou OrTs LlorCA. E' compreensivel, no entanto, acrescentamos nós, que a junção dos três arcos possa se operar, eventualmente, com certa variabilidade; assim, a fusão do 3." arco esquerdo ao tronco dos outros dois, em períodos gradativamente espaçados, e a reuniāo simultânea dos três arcos, condicionariam tôda a escala de aspectos de emergência isolada da A. carótida comum esquerda e a formação de trípode.

* No material de estudo que cstamos reunindo, cm 51 disseccōes das colaterais calibrosas do $\Lambda$ reo aórtico dêste animal, assinalamos 5 vêzes a referida disposição. 
Quanto ao Tronco bicarótico, acreditamos tenha sua gênese ligada mais ao crescimento intersticial que, habitualmente no porco e casualmente no cão. se faria com maior intensidade entre os $3 .^{\circ 8}$ arcos direito e esquerdo.

E' evidente que a preponderância do crescimento intersticial entre o $30^{\circ}$ e $40^{\circ}$ arcos direitos, por si só, prestar-se-ia da mèsma maneira a explicar a emergência separada da A. carótida comum esquerda; a fusão de tractos mais extensos do 3. e $4 .^{\circ}$ arcos direitos também conduziria a idêntico resultado. Por outro lado, a hipótese da formação do Tronco bicarótico, como decorrência da junçĩo dos 3. ${ }^{\circ 8}$ arcos aórticos, aos quais viria ligar-se ulteriormente o 4. esquerdo, embora menos provável, é perfeitamente verossímil, como aceitável é que, constituida a A. braquiocefálica, o crescimento intersticial possa determinar, secundàriamente, a concentração das Aa. carótidas.

Querer subordinar a maioria dos fenômenos apreciados, como sugere o trabalho de Orts LlorCA, à ausência das Aortas ventrais nos mamíferos domésticos, parece-nos incerto; menos objetável é que as pequenas diferenças de desenvolvimento dos arcos, tanto na fase branquial, quanto na post-branquial, condicionadas por fatôres genéticos, possam ser modificadas pela ação simultânea do deslocamento do coração, da migração arterial e da conformação do tórax.

\section{RESUMO}

Esta nota, sôbre a freqüência do Truncus bicaroticus em cães, registra a ocorrência de 5 casos de emergência das $A a$. carotides communes dextra e sirnistra, рот tronco comum, assinalados em 139 dissecções das colaterais calibrosas da aorta.

0 material utilizado compunha-se de animais adultos (72 machos, 19 fêmeas e 4 de sexo ignorado) e fetos de têrmo ( 17 machos, 22 fêmeas e 5 de sero não apurado), totalizando 139 observações realizadas, predominantemente, em animais bastardos, dentre os quais, os adultos, apresentavam idades amplamente varíiveis.

Anotada 5 vêzes, ou seja, em 4 machos (obs. 5-9-14-65) e 1 fêmea (ohs. 128), a ocorrência, apenas em um dos casos correspondia a feto de têrmo, macho (obs. 65). Em outros 3 exemplares, 1 fêmea (obs. 54) e 2 machos (obs. 104 e 135), todos adultos, processando-se a origem das Aa. carótidas comuns e subclávia direita à mesma altura, a $A$. brachiocephalica assumia aspecto de tripode. $\cap \mathrm{T}$. bicarótico estava presente também em 2 preparados, uma fêmea adulta fobs. 35) e um feto de têrmo, fêmea (obs. 66), mas, como disposição associada e conseqüente a anomalias de origem e de decurso da $A$. subclávia direita, descritas em trabalho precedente. Em grupo de 6 fetos de têrmo, provenientes da mesma cria (obs. 61 a 66), um dos machos (obs. 65) e uma das fêmeas (obs. 66) exibiam, respectivamente, T. bicarótico e A. subclávia direita como última coíateral do Arco aórtico, enquanto os mais, nada ofereciam de particular. 
A interpretação dos achados teve por base a análise do desenvolvimento dos arcos branquiais do porco (ORTS LloRCA); neste animal, como se sabe, as Aa. carótidas comuns originam-se mediante tronco comum.

Inicialmente, ao que parece, a fusāo dos $4 .^{\circ}$ e $3 .^{\circ} \operatorname{arcos}$ direitos e do $30^{\circ}$ esquerdo e mais o crescimento intersticial determinariam a formação da A. braquiocefálica. Admitindo-se que esta fusão possa se operar, eventualmente, com certa variabilidade e que o crescimento intersticial se venha a dar com maior intensidade entre os $3 .^{\mathrm{s}}$ arcos - e isto explicaria a constituicão do T. bicarótico - comprcender-se-iam as diferentes disposições que as colaterais daquela artéria oferecem. Com os mesmos elementos, outras hipóteses julgadas verossímeis foram formuladas.

\section{SUMMARY}

In this note, dealing with the frequency of the Truncus bicaroticus in dogs, 5 cases containing the Aa. carotides communes, dcxtra and sinistra, arising from a single trunk, were found on 139 dissections of the conspicuous collaterals of the aortic arch.

The material used comprised adult animals ( 72 males, 19 females and 4 of unknown sex) and full term foetuses ( 17 males, 22 females and 5 of unknown sex), summing up to 139 observations, performed mostly in mongrel animals, among which adults presented great variations in age.

The 5 cases of $T$. bicaroticus correspond to 4 males (obs. 5, 9, 14, 65) and 1 female (obs. 128); once the verification was stated in a male full term foetus (obs. 65). In 3 other adult exemplars, 1 female (obs. 54) and 2 males (obs. 104 and 135), as the origin of the common carotids and right subclavian arteries from the $A$. brachiocephalica occurred at the same level, this vessel assumed the aspect of tripod. The $T$. bicaroticus was present also in two cases (obs. 35, female, adult and obs. 66 , female full term foetus), but as an associated disposition related with anomalies of origin and course of the A. subclavia dextra, described in a previous paper. In a group of 6 foetus, one of the males and one of the females exhibited, respectively, $T$. bicaroticus and right subclavian artery as the last branch of the aortic arch; the rest presented nothing worthy of note.

The interpretation of the findings is based on the study of the development of the branchial arches in pig embryos (ORTS LLORCA); this animal offers both common carolid arteries branching from a single trunk.

The interstitial growth and the fusion of the initial tracts of the 4th and 3rd right arches and the 3 rd left one, it seems, would determine the constitution of the $A$. brachiocephalica. Admiting that this fusion may take place accidentally with certain variability and that the interstitial growth between the 3rd arches 
becomes more intense, all the different dispositions of the $A$. brachiocephalica collaterals can be traced. The same elements permit other hypothesis to be formulated.

\section{BIBLIOGRAFIA}

Bossi, V. - 1909 - "in" Bossi-Caradonna. Trattato di Anatomia veterinaria. 2. Milano, Francesco Vallardi

Bradey, O. C. - Grahame, T. - 1948 - Topographical Anatomy of the dog 5th ed. Edinburgh, Oliver and Boyd

Brandt, A. J. - 1940 - Rechtsseitige Aortabogen mit abnormen Verlauf der "A. subclavia sinistra" als Ursache von Oesophagusstenose beim Hund. Skand. Vet.: 9931105, 1940, "in" Collected Papers Vet. Inst. Stockholm. 14

Briti, A. C. - Zimmert, U. - 1947 - Trattato di Anatomia veterinaria. 2. Milano, Francesco Vallardi

Congdon, E. D. - 1922 - Transformation of the aortic-arch system during the development of the human embryo. Contributions to embriology, 14(65-71):47-110

Davies, J. J. - Ottaway, C. W. - 1943 - A peculiar case of oesophageal obstruction in the dog; congenital abnormality of the aortic arch. Vet. Rec., 55(8):102-3

Ellenberger, W. - Baum, H. - 1891 - Systematische und topographische Anatomie. des Hundes. Berlin, Paul Parey

Ellenberaer, W. - Baum, H. - 1932 - Handbuch der vergleichenden Anatomie der Haustiere. 17 Aufl. Berlin, Julius Springer

Favir.L, N. - 1931 - Nozione comparate di Anatomia e Fisiologia degli animali rurali. Torino, Unione Tipografico Editrice Torinese

Golub, D. M. - 1931 - Die Entwicklung des Aortenbogens und der von ihm ausgehenden $\ddot{A}$ ste bei Säugetieren. I: Entwicklung der Kiemenbogen bei Kalb und Menchen $Z$. Anat., 95:447-72

González y Garcia, J. - Alvarez, R. G. - 1929 - Anatomia comparada de lus animales domésticos. 3." ed. Zaragoza, "La Académica"

Lesbre, F. X. - 1923 - Précis d'Anatomie comparée des animaux domestiques. 2. Paris, J.-B. Baillière et fils

Lucarelli, F. - 1893 - Compendio di Anatomia comparata. Napoli. "in" Mobilio, C.: 36

Mannu, A. - 1930 - "in" Zimmerl - Trattato di Anatomia veterinaria. 2. Milano. Francesco Vallardi

Martin, P. W. - 1923 - Lehrbuch der Anatomie der Hausticre. 2 Auf. 4. Stultgart, Schickhardt \& Ebner

Millen, M. E. -- 1947 - Guide to the dissection of the dog. Michigan, Edwards B rothers, Inc.

Miller, M. E. - 1947 - Informação pessoal

Mosilıo, C. - 1910 - Mancanza del tronco brachio-cefalico commune in un "Equus mulus". Arch. Sci. R. Soc. Naz. Vet., Torino, 8(3-6):33-44

Orts Llonca, F. - 1934 - Uber die Entwicklung der Arterienbogen beim Schweine. Z. Anat., $102: 335-47$

PaIva, O. M. - 1948 - Dois casos de "A. Subclavia dextra" como últimn colateral do “A rcus aorticus" no cão. Rev. Fac. Med. Vet., S. Paulo, 3(1):203-22

Sisson, S. - Grossman, J. D. - 1945 - The Anatomy of domestic animals. 3rd ed. Philadelphia, W. B. Saunders Company

Stieve, H. - 1939 - Nomina Anatomica. 2 Aufl.: 144. Jena, Gustav Fischer 